by pressure, was not so materially affected by it as that on the opposite side. There was pain referable to the vertex, with palpitation of the heart, though the sounds were normal. Her appetite was good, and the functions of secretion and excretion were naturally performed. Upon swallowing, the tumour moved as in ordinary bronchocele : there was no dysphagia, and the dyspncea was very trifling, and only complained of at night, though the voice was somewhat modified in tone, and rather shriller than natural.

Treatment.-Viewing it in the light of a true aneurism, she was at first put upon spare diet, consisting principally of milkand-bread; pressure was applied topically, first by Weiss's compress artery, and with apparent relief, but owing to the weight and the inconvenience it caused the patient while lying down, it was obliged to be removed; subsequently an elastic band, composed of vulcanized indian rubber, was sub. stituted, affording evident relief. The diet was afterwards changed to a more generous one, and occasional doses of aperient medicine given. Under this treatment the patient continued for six weeks, when she was discharged as an outpatient. I have since had the opportunity of seeing her several times, and although she was not cured, I think some diminution in the tumour has taken place, and her health remains exceedingly good.

Diagnosis. - That the disease was that of aneurism by anastomosis, I am led to affirm-first, from the peculiar feel it communicated to the touch; secondly, from the appearance of the skin covering the tumour, and a pecrliar twist which was heard; and lastly, that upon firm pressur: the tumour almost subsided. That it was not a scrofulous tumour, which is not unlikely to be confounded with this disease, I infer from the constitutional symptoms present, the absence of any inflammatory symptoms setting at rest the question of an abscess with a large artery situated behind it.

Prognosis.-The prognosis in this case, of course, as in most cases where the tumours are situated in or about the neck, on soft parts, or are deeply seated, and one of considerable size, and where neither delegation nor compusition are capable of being applied, must necessarily be unfavourable; again, when the disease is complicated, as in the present instance, with the same existing pulsatory tumour in the orbits, threatening on the one hand apoplexy, and on the other hypertrophy of the heart, the difficulties to be encountered by the surgeon are rendered both manifold and tedious.

\section{NEWCASTLE.ON-TYNE INFIRMARY.}

Reported by A. TAYLOR, Esq., Dresser to Sir John Fife.

REMOVAL OF AN ELASTIC TUMOUR FROM THE UPPER JAW.

ON Thursday, the 9th of November, 1848, John F-, furrier, from Sunderland, presented himself at the Newcastle Infirmary with his face disfigured by a remarkable projection of the upper jaw. An elastic tumour occupied the palate, and filled the mouth; it was attached to, and arose from, an extensive surface of the inner part of the upper jaw, from the last molar tooth on the left, to the middle of the right side. The alveolar process was everted as far as the attachment of the tumour. He was operated upon on Tuesday, the 14th. The operation was performed as follows:-

Sir John Fife, after administering chloroform, made incisions from each angle of the mouth to the lower and outer side of each orbit; tied both facial arteries, and then dissected up a flap, exposing the upper jaw from the left pterygoid plate of the sphenoid bone to the middle of the right maxillary bone. A small saw was then applied to the pterygoid plate of the sphenoid bone on the left side, carried nearly horizontally across above each canine fossa, and quite through, exposing the left antrum and both nerves. The third molar tooth on the right side was then extracted, and the bone above it divided by Liston's forceps. Three-fourths of the upper jaw were thus detached along with the tumour, excepting. where the membrane held its posterior part to the palate. Introducing a probe-pointed bistoury on the finger, down the pharynx, the membrane was divided from behind forward, and the whole mass brought away. The saw having taken off a horizontal section of the top of the tumour, Sir J. Fife found some of it in the nostrils, loose, and some in the left antrum adhering. These were removed, and the antrum was canterized. The flap was then brought down, and secured by sutures. The patient was much exhausted, but the face was no longer distorted.

November 17th.-The patient is daily improving.
OBSERVATIONS ON

THE PATHOLOGY AND TREATMENT

or

GONORRHEA IN THF FEMALE。

By JOIIN C. EGAN, M.D., M.R.I.A.,

FELLOW OF THE ROYAL COLLEGE OF SURGEONS IN IRELAND, LATELY ONE OF THE SURGEUNS TO THE WESTMORELAND LOCK HOSPITAL.

As a warm admirer of $M$. Ricord, and conceiving that his investigations had been pursued in a spirit of true philosophic research, unbiassed, on the one hand, by prejudice, and unfettered, on the other, by preconceived opinions, I was determined, while opportunity was afforded me, to test for my own satisfaction the accuracy of the experiments, the results of which are recorded in his work on venereal diseases. With the soundness of his researches on inoculation generally, corro. borated by the uniformity of the results which I was enabled to confirm, I have been extremely gratified, and have since been led to look upon that process as forming a valuable adjunct in the differential diagnosis of syphilitic affections: many of these experiments I have subsequently had the pleasure of seeing confirmed by M. Ricord himself, in the wards of the Hopital du Midi, at Paris.

The prevalence of gonorrhoea in Dublin, during the last few years, has offered unusual advantages to a person anxious to investigate the true nature of that disease, and in no institution were greater facilities afforded for such a line of study, than in our Lock Hospital, containing 130 female patients, two thirds of whom, at least, were at one time labouring under blennorrhagia. I was the more desirous of making myself perfectly familiar with the appearances of the vagina and uterus, as presented by the speculum, as no British hospital had, up to that period, contributed any information in its pathology or treatment, and the only data for our guidance were supplied from continental institutions; added to which, medical men of the highest repute and most extensive midwifery practice seemed unable to afford me, from their multiplied cases, the information I too often required. In pursuing those investigations, I was particularly struck with the absence, in the majority of instances, of a condition of the uterus described by writers of the French school, as "granular erosion" of the neck of that organ, a lesion which is stated by $M$. Ricord to be discernible in every nineteen out of twenty cases of gonorrhœa, thus enabling him to form a differential diagnosis between the infectious and the more common forms of vaginal discharges to which females are subject. This appearance only presented in thirty-eight out of 112 cases, which I submitted to examination; I was therefore led to conclude that from the absence of this symptom of disease in women, where no doubt could exist as to the venereal origin of the affection; and, on the other hand, from its occasional presence in females labouring under leucorrhcea, whose rank of life and virtuous habits place them beyond all suspicion of infection; that we should not be justified, in this country at least, in pronouncing, either in a medico-legal point of view or otherwise, from evidence furnished from this source, the true nature of the complaint. This, I contend, is a point of paramount importance: either the disease assumes a different character, and produces different local effects on the continent, or $\mathbf{M}$. Ricord must have fallen into an error that, to the medical jurist, might have been productire of grave effects, and biighted for ever his professional reputation. How such a statement could have remained unrefuted till now, I cannot for a moment conceive; except on the supposition of want of sufficient opportunity to decide the question! Again, in following up these investigations, it was a matter of no less surprise to me to find, in opposition to the recorded opinions of others, that the disease, in the majority of cases, extended to the lining membrane of the uterus, which was sufficiently demonstrated by a muco-purulent discharge fiom the os. Here it may be a fitting occasion to notice a remark made by Dr. de Meric, that both Dr. Mayne and I had been anticipated by $M$. Ricord, as regards the participation of the uterus in the disease. I would beg leave to renind Dr. de Meric, that on no occasion did I lay claim to priority in the discovery of that fact; as $I$ was aware that $M$. Ricord had, in a few desultory remarks, alluded to the participation, in protracted cases, of the womb in the disease; it appears to me, however, that any person who will take the trouble of reading the following short paragraph, which I transcribe from his work on venereal diseases, (Drummond's translation,) will at once perceive that he is describing a very different affection from that which $I$ have 
stated occurs most frequently as a concomitant symptom of the discase:-

"Like other portions of the mucous membrane of the sexual organs, the internal surface of the womb is frequently the seat of ulcerations which the means hitherto pointed out cannot cure. We must here, as in the ulcerations of other parts, modify the surfaces in a more powerful manner, but the greatest precautions are necessary in cauterizing the interior of so delicate an organ, the reaction of which would be so powerful; for while the most powerful caustics applied to the orifice of the cervix generally produce no pain, fluids scarce possessing any caustic properties, being introduced into the cavity of the uterus, may cause the most serious consequences." --p. 327.

This peculiar form of disease, if ever it does occur in blennorrhagia, must be extremely rare, else how could we account for the persistence of the complaint for a series of years, withont producing that disorganization which we know is consequent upon ulceration, when confined to other important tissues, - the bladder, for example,-and which rarely fails in producing well-marked symptoms of an alarming character on the constitution generally, and not unfrequently perils the life of the sufferer. Surely pain would be a prominent symptom, were an organ of such importance as the uterus engaged in ulceration, and yet such an effect is rarely complained of, even when the disease has gone on for years. We might likewise expect that an occasional loss of blood would result on such disorganization, but I do not remember having ever met with a single instance where such a symptom was present. It must therefore, I think, be evident to any unprejudiced mind, that the affection which I have described as so frequently observable in patients suffering from blennorrhagic discharges, is different from that adverted to by $\mathbf{M}$. Ricord, in the passage just cited, and consists, not in ulceration, as I had more than once an opportunity of witnessing on post-mortem examination, but on a chronic inflammation of the lining membrane of the uterus. But in order to prove M. Ricord's priority of claim, Dr. de Meric has referred to his twelfth leeture, "delivered in the summer of 1847 , and published in THE LANCET of the 5th of February last, where he states that "it is now proved that blennorrhagia may reach the uterus, and even run along the Fallopian tube to the ovary." In these few words, I take it, are contained, though not fully expressed, M. Ricord's true explanation of the pathology of the disease. As he makes no allusion to that ulceration of the interior of the womb which on a former occasion he laid down as giving rise to blennorrhagic uteri, I think we may conclude that he is now describing-when he states, "blennorrhagia may reach the uterus, and even run along the Fallopian tubes to the ovary"-inflammation upon which this discharge is consequent, and which $I$ have endeavoured to show is the true condition of the parts under such circumstances, and not ulceration, in which $I$ believe the disease rarely terminates. Most willingly, therefore, while I agree with Dr. Mayne that "this must be held to be the real date of his opinion above quoted becoming known to the profession in England," do I concede to M. Ricord the honour of priority as claimed by his translator, although this lecture did not appear in print till my paper (which was read before the Surgical Society of Ire. land, on the evening of the 18th of March) had been prepared and substantiated by cases. As regards the treatment of the disease in my hands, copaibal injections have proved completely abortive in arresting the progress of the disease, and I think, in the treatment of this, as well as of every other affection, we are not justified in drawing deductions from a few isolated cases, which on a larger scale might probably but form exceptions to the general rule. I may likewise add, that the idea of the modification of copaiba by renal elaboration, as suggested by Dr. de Meric, is not, I apprehend, so visionary or preposterous as Dr. Mayne would seem to insinuate, and it is to that opinion $T$ have long since leaned, in consequence of the want of success which attended, in my practice, its local application.

Since forwarding the foregoing observations, I have had an opportunity of perusing Dr. Mayne's remarks, as contained in The Lancer for October 21 st, to which I would reply a few words. "I hope (to use the language of Dr. de Meric) that, in performing my task, I shall never so far forget the dignity of our profession, as foolishly and pettishly to attack any member of it." Passing over, then, the style and tone that pervade Dr. Mayne's remarks, I will remind the reader that in a former communication Dr. Mayne stated " that he found the disease (blennorrhagia) obedient to the use of these (copaibal injections) more than of any other kind of injection usually employed:" he now goes on to illustrate the efficacy of the copaibal solution, by reference to two cases of gonorrhoeal ophthalmy.

Dr. Egan recapitulates the cases, which will be found at p. 475 , and he then remarks:-

In furnishing cases to exemplify an author's ideas, or illus trate his views on any particular subject, it is usual to state, as precisely as possible, every circumstance, both as regards the symptoms of the disease, and the line of treatment adopted. In this country, fortunately, gonorrhceal ophthalmia does not very frequently present itself to our notice, on which account it is a matter of some importance that we should be made acquainted with every circumstance in a disease so interesting to the surgeon and pathologist, at the same time so destructive to the sufferer, the more so when a specific is proposed for its cure. Now has this been the case in the two instances before us? We read of a child, six years of age, (which, by the way, is rather unusual,) being affected with gonorrhoeal vaginitis, and at the same time labouring under gonorrhœeal ophthalmia, having both diseases cured by one and the same remedy, the strength of which is never even hinted at, leaving us in doubt as to whether the same proportion of balsam of copaiba was employed in the formation of the vaginal injection as in the collyrium. It is true Dr. Mayne has told us, in a former communication, that "about a drachm of the soluble balsam to a pint of water will be found generally applicable," but whether this proportion is to be increased or diminished, in ophthalmia of a child six years old, we have no means of ascertaining. Nor are we informed as to the manner in which the ophthalmic complaint was communicated to the child, whether by direct contact, or metastasis, as Dr. Mayne must be well aware that, even at the present day, there is a considerable diversity of opinion on this very point, which, had he been more precise, he might have assisted in reconciling. The same uncertainty attends the length of duration of the disease, having been only informed that, previous to her coming under Dr. Mayne's care, she went the round of all the quacks \&c. for many weeks, which, I need scarcely say, may mean either five, seven, twelve, or an indefinite number of weeks. Now, so far as my experience of gonorrhœal ophthalmia extends, I must confess that $I$ never saw a case where active means were not resorted to in the very onset, run on for many weeks without terminating in the destruction of the entire structure of the eye, and in this opinion I am fully borne out by Lawrence, Mackenzie, Wardrop, Delpech, Ritcher, Scarpa, Jaceb, and many other equally respectable authorities that might be adduced. "The first thing to be urged in the treatment of gonorrhoeal ophthalmia is prompt and energetic employment of the treatment, as hesitation involves most frequently the loss of the eye." (Ricord.) "Not a moment should be lost in endeavouring to control the disease, and our treatment in catarrhal syphilis of the eye must be more energetic than in any other form of the disease." (Wallace.) "Gonorrhoeal ophthalmia is perhaps the most violent and destructive spocies of inflammation to which the eyes are subject, and if not met promptly by active and appropriate measures, the organ will soon be destroyed." (Carmichael.) The gonorrhœal is generally more rapid in its progress than any of the other varieties of iritis, and is one of the most severe and formidable whilst it lasts." (Mackenzie.) "Anti-gonorrhceal medicines, as copaiba, cubebs, \&c., have no action on this disease, in whatever manner they may be administered," (Ricord.)

The description of the second case of gonorrheal ophthalmia quoted by Dr. Mayne is, if possible, more imperfect than the first; we are told nothing of the length of standing of the disease, nothing of the strength of the copaibal solution, how often it was repeated, and this, too, in a case "where congestion was excessive, and chemosis complete." "The copaibal solution was applied, and in a few days the appearance of the eyes was much improved, but inflammation by this time (increased, $I$ suppose, by the irritating quality of the copaiba) became evident in the sclerotic coat and iris. The necessary treatment was instantly put in practice with success, and by then persevering with the copaibal application alone, the cure of the gonorrhoeal inflammation was in four or five weeks completed." Here, we.are informed that the use of the solution was obliged to be dispensed with, and the "necessary treatment," by which, I presume, is meant the usual treatment resorted to in inflammation of the eye, "was instantly put in practice with success;" and here the case closes without any allusion to the gonorrhoea under which the man laboured, or any reference to the efficacy of copaibal injections, which in this instance, I imagine, was tested. Upow the foregoing 
cases I forbear any further comment. In the next paragraph, Dr. Mayne announces a physiological discovery, "that gonor" rhoa in the female, when the uterus is implicated, must inevitably prove an obstacle to conception, according to the generally received and most intelligible theory of that mysterious act." And here I must again regret, that previous to committing his observations to the press, Dr. Mayne had not an opportunity of reading my paper, to which I before referred him, as he might have there perceived that he has not been the first to observe that " barrenness is attributable to this, and to similar easily comprehended causes, much more frequently than has ever been thought of." In describing, in that essay, the appearnees presented on speculum examination, I have made the following observations:-

ss The most common appearance $I$ have met with is that which $I$ have described as an erythematous condition of the cervix, generally accompanied with engorgement and slight induration. This was in many cases concealed from view by the intervention of a thin, semi-transparent stratum of mucopurulent matter, with which the inflamed cervix was slightly coated. This condition was almost invariably accompanied with a discharge from the interior of the uterus, of a very tenacious and almost purulent matter, forming, in this respect, a marked difference between this secretion and that furnished by the vagina and exterior of the uterus. A string of this ropy material was generally found suspended from the os tincæ, which was with considerable difficulty disengaged, and removed by the assistance of a sponge. This, together with menstrual irregularity, will, I doubt not, in a great measure, account for the almost universal sterility common to this class of females. The same disposition has been observed by Messis. Gendrin, Jobert, and Emery, in young married women labouring under lencorrhœa."

Dr. Mayne might not only have perceived that I had anticipated him upwards of seven months ago in that opinion, but that the authorities just cited had observed a similar coincidence in young married females labouring under leucorrhoa, and who had never been exposed to gonorrhoal infection.

Passing over some of Dr. Mayne's other remarks, I arrive at an announcement, for which, I must confess, I had been hitherto unprepared by hospital experience, private practice, or a course of study, to which I have been accustomed for several years: "That a sound uterus, under certain conditions, gives out a thick, yellow mucus, so like to the mucopurulent discharge from the same organ, when affected by gonorrheal inflammation, that the distinction is very difficult to be made." Surely Dr. Mayne does not mean to affirm that a diseased action can go on in a healthy structure, of which a thick yellow mucus is undoubtedly the result? If so, all my ideas of pathological anatomy have been hitherto founded on erroneous principles, and the sooner the opinions of Corvisart, Bayle, Laennec, Broussais, Lallemand, Andral, Baillie, and Bright, have been supplanted by those of Dr. Mayne, the better for continental and British pathologists. Dr. Mayne objects to the term I have made use of to designate a perfect restoration to health-viz., a permanent cure, and contends that if there be a cure at all, it must of necessity be a permanent one. Has it ever fallen to Dr. Mayne's lot to treat any of the secondary or tertiary forms of syphilis? if so, he can be at no loss, $I$ conceive, to comprehend my meaning. What is more common than in the papular, scaly, pustular, and phagedænic forms of syphilis, after every symptom of the disease has disappeared, than to have it return after a few weeks, or months, the patient in the interval having enjoyed perfect immunity from any form of the complaint? Or is it not of every day occurrence, in syphilitic ulceration of the throat, after a cure has been pronounced by the medical attendant, and the patient has congratulated himself on perfect restoration to health, to find the same symptoms return, and the same appearances present, perhaps in a more inveterate form, after the lapse of months? Does not the same occur in syphilitic periostitis? and have we not too often to lament a like, but more frequent, consequence of gonorrhcea in the female? These are cases of what I term apparent, in contradistinction to permanent cure, where a latent venereal taint is indicated by the recurrence of the disease, and which is alluded to by M. Ricord when he says," If we calculate the cure of a chancre from the day the ulcer has cicatrized, without troubling ourselves about what may take place after, it will be sometimes apparently quicker by the simple treatment without mercury." By Mr. Parker, who observes, "When a certain degree of induration accompanies a chancre, or persists after its apparent cure, recourse

- Dublin Quarterly Journal, May, 1848. may be had to mercury." By Mr. Carmichæl, when he states, 'I have seen, even in this exhausted state of the disease; (the phagedænic form, " more relapses than perfect cures by mercury." By Mr. Colles, when he affirms, "I need not repeat the admonition of taking care to excite the mercurial, action in the manner most likely to ensure its sanitary operation, to produce the least possible injury to the constitution, and yet to excite and keep up such a mercurial action as we know is most likely to effect a permanent cure of the venereal disease." I have now, I trust, sufficiently explained to Dr. Mayne's satisfaction, the sense in which i have employed the term "permanent cure," and he will, I think, be disposed to agree with me, that in this sense I have not adopted it, without sufficiont precedent from writers of no mean authority on syphilitic affections. Dr. Mayne suggests that there may be other reasons for finding, at some future. period, the os uteri swollen and patulous, giving exit to a vitiated secretion, \&c., "than persistence of a first and only infection." In this sentiment I fully concur with Dr. Mayne and he is much mistaken if he imagines that I consider a first attack of gonorrhœa, like some exanthematous diseases, renders the patient proof against a second infection; but the class of females to whom I particularly alluded, as being the subject of relapses, were those who, in my original paper, I stated had determined to abandon a life of misery and profligacy, and with such intention became inmates of some of those asylums or penitentiaries with which our city abounds. It would be a vain waste of time to enter into the particulars respecting the strict moral discipline and admirable surveillance with which these excellent charitable institutions are conducted. Suffice it to say, that the discipline there observed must be a sufficient guarantec against any chance of renewed infection, and it must be therefore at once obvious, that patients returning to. hospital with symptoms such as I have enumerated, must be the subjects of relapse, and not the victims of renewed infection. These relapses, I have stated, had been brought on by bodily exertion, as washing, \&c., and appeared to have been the result of a general relaxation of constitution, in which a tonic line of treatment was obvionsly indicated. In these cases, on speculum examination, the os uteri was found swollem and patulous, giving exit to a vitiated secretion. I find I have inadvertently misquoted Dr. Mavne in stating, that as a substitute for the solid nitrate of silver he proposes an ointment of six or eight grains of nitrate of silver to an ounce of lard, which should have been a "drachm of lard," and I am happy to have this opportunity of apologizing tohim for this misinterpretation. My views, however, upor this subject remain as fixed as formerly, and no additional argument has been adduced, which would lead me to change the opinions I have before expressed. As Dr. Mayne still appears apprehensive lest injury might ensue from the breaking of the caustic pencil, I have only again to refer him to my original paper for a plan by which even that casualty may be obviated. I have never met with any cases, as described by Dr. Mayne, whete "hysterics and fainting" resulted upon theapplication of the caustic to the vagina, althongh such symptoms have been recorded as consequent upon the injection of fluids into the cavity of the uterus itself; on the contrary, as I mentioned in a former communication, in order to illustrate the trifling uneasiness produced by this remedial agent, " that patients are constantly in the habit of walking distances to their homes, almost immediately after the operation." As regards the administration of the balsams internally, Dr. Mayne seems to think that $I$ have now modified my opinion as formerly expressed, by now writing of their inutility; this, however, is substantially the idea which I have before recorded, and Dr. Mayne had no authority for implying, that by the use of that phrase I meant their " total" inutility, having been well aware that they have been said to have proved successful in cases where the urethra alone had been affected, but knowing that those instances are of very rare occurrence, indeed, I did not think it judicious to direct special attention to them. As regards the use of copaibal injections I beg leave to state, in answer to Dr. Mayne's queries, that not wishing to lose valuable time, or prolong the patients' confinement in the hospital, thereby inflicting upon them a cruelty for which I should have been highly culpable, I did not persist with the administration of these injections beyond a few months, which, I regret to say, was even too lengthened a period than that required to demonstrate their utter fu ility. I may likewise add, that as my communications were principally read before the Surgical Society of Ireland, I did not consider that I should have been justified in consuming the time of the meeting, to the exclusion of the discussion of more important topics, by detailing the remedies, (which, indeed, were not a 
few, that in $\mathrm{my}$ hands proved successful in controlling this affection; there is therefore no published account of the failure of copaibal injections in gonorrhœa, previous to my last communication in this journal.

$I$ have now endeavoured to reply scriatim to Dr. Mayne's statements, and to answer, to the best of my humble ability, his different objections. If I have, by any unhappy expression, given the slightest umbrage to any, I beg, in conclusion, to state that such a course has been quite foreign to my intention, and that I have all along endeavoured to apply myself to statements, not to pass strictures upon one, who, for aught I know, may be far beyond my praise or censure.

Harcourt street, Dublin, Nov. 1848 .

-

\section{REMARKS ON}

\section{THE PHYSIOLOGY OF THE CHORDAE VOCALES.}

\section{By J. D. MACDONALD, Eso., London.}

Wттногт entering minutely into the anatomy of the larynx, it will be necessary, at the outset of my remarks, to give a brief sketch of the chordee vocales, the organs to which the following observations particularly refer.

Within the larynx, and on either lateral wall, two folds of lining membrane may be seen, one lying superior, and the other inferior, while both extend backwards, diverging from the angle of the thyroid cartilage to the fore part of the arytenoid of their respective sides. The lower folds enclose a well-defined, cord-like mass of yellow, fibrous element, which is not so distinct in the upper, and in consequence of this difference the former have been termed the "true," and the latter the "false cords." By the action of certain muscles upon the moveable cartilages of the larynx every degree of tension is effected in these cords which the case may require. Between the chordse of orposite sides is the opening of the rima glottidis, and between the upper and lower cord of each side is a deep pit, the ventricle of the larynx, which allows of their free vibration. The lower or true cords are alone acknowledged by physiologists to vibrate in the formation of the voice, while the superior, or (so-called) false, are presumed to be passive; but if the subject be properly investigated, there will appear no sufficient reason to exclude them from a share in this function. The lower cords enjoy a certain range of vibration, which we may limit to one octave, the compass of the natural voice; but for the production of notes extending beyond this sphers, it is only reasonable to suppose that the upper cords are, in their turn, brought into play; and thus an explanation of the falsetto, or feigned voice, is afforded, the peculiar quality of which is owing, no doubt, to the structure of these cords, their delicary being essential for treble articulation, whereby they are fitted to continue the chromatic series from the point where the capacity of the true cords cease. And here we are furnished with a further explanation as to the cause of the breach that takes place in the juvenile voice on approaching manhood; for where the tension of the upper cords is not effectually applied, in continuing the scale, and the lower ones fail to produce their usual sounds, in consequence of their elongation, (by the prominent development of the angle of the thyroid cartilage, the transition of vibrations from the lower cords to the upper becomes abrupt, and the breach is manifest in the voice. But by the more equal development of the cords, at a subsequent pericd, this defect ceaser; although still the quality of tone of the superior cord is sufficiently distinct from that of the inferior to characterize the fulsetto, or artificial voice, as contradistinguished from the natural voice.

In the youthful voice, before the complete development of the thyroid cartilage and other parts of the larynx, accompanied by an elongation of the cords, the transition of vibrations from the lower to the upper cords is so delicate and insensible, that the scale may be articulated perfectly throughout without any apparent difference in quality of tone. But when the change above mentioned takes place, the cords are for some time incapable of articulating the notes peculiar either to the youthful or adult voice, until, to the maturer development of the parts, a power of lung is superadded with the growth and accumulating strength of the individual. Previous to these changes, the juvenile voice is essentially treble, extending from $\mathrm{C}$ below to $\mathrm{G}$ ahove the stave; while the ordinary tenor can reach no farther than $D$ on the fourth line (treble) by the simple exercise of the natural voice; but by what is termed the falsetto, or artificial voice, many higher notes may be accomplished. The anatomical construction of these organs singularly confirms the above considerations, as an acute sound can no more be emitted by a base string than can a low note be produced by a treble string.

Assuming these points to be correct, the difficulty of connecting the functions of the lower with those of the upper cords is sufficiently obvions, from the practice which is required to effect an even transition from the natural to the artificial voice.

The term "false," as applied to the upper cord, seems to have deterred physiolugists, hitherto, from looking to these organs for an explanation of a function which they find some difficulty in accounting for. (The term falsetto cords would be more appropriate.) In the alssence of such experimental researches as might demonstrate the above opinions to be true, an inspection of the larynx even of the lower animals will instantly show that the upper cords are not so insiguificant as their name would imply.

The chordic vocales no doubt combine the characters of the vibrating cord with those of the reed; and thus the peculiar quality of tone in the human voice is accounted for. Each cord enveloped by the lining membrane of the larynx presents a horizontal fold, having a free vibratile margin internally, and fixed by its outer border and extremities. This arrangement does not in any way linder its motion: indeed, this may be practically shown by procuring a sheet of India-rubber, and making tense a small portion of its margin between the fingers, when, by blowing upon it with the breath, a very clear musicsl sound will be emitted, which will become more acute, or grave, as it is made tense or relaxed. The varied powers of imitation possessed by the human voice, in assimilating natural sounds, is no doubt attributable to its combining, in the structure of the larynx, the properties of both a stringed and wind instrument, to the nnion of which the sweetness and power of the vox liumana is mainly owing. Newman-street, 1848 ,

\section{ON A}

REMARKABLE CASE OF OCULAR DISEASE PRODUCED BY AN ELECTRIC DISCHARGE. Bx THOMAS CUTLER, M.D.,

PHYSICIAN TO THE DISPENSAIRE GENERAL AT SPA, BELGIUM.

I was consulted, a few days since, by Mons. I-, a lientenant in the Belgian Custom-house service, for a defect of vision which, at the first moment, I considered to be only a case of advanced amblyopia; but by a more minute examination, I was led to the discovery of the following interesting circumstances:-

Mions. I_- residing at Viel-Salm, on the Prussian frontier, fifty-one years of age, of tall stature, of a sanguineo-nervoustemperament, not stout, but of a well-knit frame, and whohad been all his life in the enjoyment of robust health, with a remorkably keen vision, was, about six weeks ago, while out on duty, overtaken, in the neighbouriug mountains, by a terrific thunder-storm. On the occurrence of a more than usual vivid flash of lightning, followed almost instantaneously by a violent peal of thunder, Mons. L- felt as if struck by a violent blow on the head, his vision became dim, and he immediately set about returning home. Before reaching his house, the head began to swell to that degree, that upon his arrival he became much alarmed, and sent for the surgeon of the neighbourhood. He describes the swelling to have been equally diffused over the entire skull. He was bled, leeched, several times cupped on the nape of the neck, and otherwise treated antiphlogistically; under which means the tumefaction gradually subsided, and at the end of a fortnight the soft parts returned to their normal condition. The amblyopia continuing, however, to increase, his medical attendant recommended him to visit Spa, and consult me forthwith. I found the pupils much dilated, sluggish in their contraction even before a powerful light, but no change in the transparent humours. Upon closely questioning him, I ascertained that he had become nyctalopic-objects appearing before sunset enveloped in a thick haze, while from that time until morning they were again seen quite distinctly. Suspecting at least a faint central opacity of the lenticular system, I examined him still more attentively by the aid of a loupe $a$ diaphragme; but failing to discover any pathological alteration, I subjected him to the well-known test of Purkinje. The result was the same-the refractive structures of the eye were perfectly transparent, and there could be, consequently, no further doubt of its being a true case of nyctalopia.

Willing to ascertain if there were any other phenomena connected with this defect of vision, I tested him upon the 